\section{Estimación del efecto marginal de la vegetación urbana en la remoción de PM2.5 en Medellín, Colombia.}

\author{
Daniela Velásquez Ciro' ${ }^{1}$, Julio Eduardo Cañón \\ Barriga e Isabel Cristina Hoyos Rincón \\ ${ }^{1}$ Universidad de Antioquia, Colombia \\ daniela.velasquezc@udea.edu.co
}

\author{
Estimation of the marginal effect of urban \\ vegetation on the removal of PM2.5 in Medellín, \\ Colombia.
}

\title{
RESUMEN
}

En la actualidad, la ciudad de Medellín, en Colombia, tiene un problema crónico de calidad de aire. Una alternativa para reducir los efectos de esa contaminación es aumentar las áreas arborizadas. En este trabajo se estimó el efecto marginal que tendría el aumento de la cobertura arbórea urbana en la remoción de PM2.5. En este sentido, el mismo hace uso de la metodología UFORE para calcular la remoción de este contaminante en forma distribuida sobre la ciudad, utilizando imágenes satelitales Sentinel 2A para identificar las coberturas y estimar el Índice de Área Foliar (IAF), que es un insumo importante para los cálculos. Se tomaron como base los registros de estaciones en el año 2017. Al calcular la remoción del PM2.5 para la condición base, se encontró un valor cercano a 83 t para toda el área metropolitana y de $3 \mathrm{t}$ para Medellín. Cuando se aumenta el área total arborizada en un $1 \%$ de manera proporcional en toda la ciudad, el valor de la remoción aumenta en $1 \%$. Por otro lado, al localizar la arborización en las zonas de mayor concentración del contaminante, la remoción total tiene un aumento cercano al 1.6\%, lo que implicaría que la decisión de arborización debe priorizarse en dichos lugares para que sea efectiva. Igualmente, se estimó preliminarmente que optimizar el IAF de la cobertura arbórea puede resultar más efectivo en la remoción que aumentar el área de arbolado urbano, aunque otros beneficios de los árboles deben tenerse en cuenta para las decisiones de siembra.

PALABRAS CLAVE: cobertura arbórea, contaminación atmosférica, modelo UFORE.

\section{ABSTRACT}

At present, the city of Medellin, in Colombia, has a chronic air quality problem. An alternative to reduce the effects of such contamination is to increase tree-lined areas. In this work, the marginal effect of the increase in urban tree cover on the removal of PM2.5 was estimated. In this sense, it uses the UFORE methodology to calculate the removal of this contaminant in a distributed manner over the city, using Sentinel 2A satellite images to identify the coverage and estimate the Foliar Area Index (IAF), which is an input Important for calculations. The records of stations in 2017 were taken as a base. When calculating the removal of PM2.5 for the base condition, a value close to $83 \mathrm{t}$ was found for the entire metropolitan area and $3 \mathrm{t}$ for Medellin. When the total tree area is increased by $1 \%$ proportionally throughout the city, the value of the removal increases by $1 \%$. On the other hand, by locating arborization in the areas with the highest concentration of the pollutant, the total removal has an increase close to $1.6 \%$, which would imply that the arborization decision must be prioritized in those places to be effective. Likewise, it was preliminarily estimated that optimizing 
tree cover IAF may be more effective in removal than increasing urban tree area, although other tree benefits should be taken into account for planting decisions.

KEYWORDS: air pollution, tree cover, UFORE model.

\section{INTRODUCCIÓN}

La ciudad de Medellín es el municipio principal del área metropolitana del Valle de Aburrá, que incluye a los municipios de Barbosa, Bello, Copacabana, Envigado, Girardota, Itagüí, La Estrella, Medellín y Sabaneta. El Valle..., que tiene un área aproximada de $1552 \mathrm{~km}^{2}$ y una población aproximada de 3.9 millones (Agudelo y Mirallesa, 2015), se ha urbanizado indiscriminadamente con el paso de los años, aumentando las emisiones contaminantes de actividades masivas como el transporte automotor y de la industria (Área Metropolitana del Valle de Aburrá, 2019). Además, las condiciones orográficas y meteorológicas generan un proceso de estabilidad atmosférica, debido a la inversión térmica que evita la dispersión y el ascenso de contaminantes, en épocas de transición entre temporadas secas -febrero, marzo, abril- y de lluvia -septiembre, octubre, noviembre (Herrera, 2015; Rendón et al., 2014; Sistema de Alerta Temprana de Medellín y el valle de Aburrá, 2019; Área Metropolitana del Valle de Aburrá [AMVA] y Universidad Pontificia Bolivariana [UPB] (2018).

Desde 2012, las concentraciones de PM10 y PM2.5 en el Valle... han aumentado a niveles por encima de los permisibles para la salud humana, lo cual ha llevado a declarar "contingencias ambientales» por parte de la Autoridad Ambiental desde el año 2016 para toda el área metropolitana (AMVA, 2019; Contraloría General de Medellín y Universidad Nacional de Colombia, 2018). Los picos de contaminación atmosférica que han tenido lugar en los primeros semestres de 2015 a 2019 han motivado la movilización social y el desarrollo de medidas de contingencia y planes a largo plazo para la gestión de la calidad del aire en la región (AMVA, 2019).

Las plantaciones de árboles a gran escala en zonas urbanas, además de dar respuesta a necesidades sociales (de bienestar, recreativas, estéticas), tienen un efecto potencial en la intercepción y eliminación de contaminantes atmosféricos (Reynolds et al., 2017; Selmi et al., 2016), así como en la protección contra los rayos ultravioleta, la mitigación de las islas de calor, la disminución de la escorrentía, la reducción del mantenimiento del pavimento y la reducción de los niveles de ruido del tráfico (Jeanjean, et al., 2017; Rogers, et al., 2015).

Como parte de estas medidas se ha creado un proyecto para emplazar un cinturón verde alrededor de la zona urbana, en virtud de evitar su crecimiento (Agudelo y Miralles, 2015). Igualmente, la Alcaldía de Medellín y el Gobierno Nacional han considerado diferentes medidas para reducir la contaminación del aire, entre las que se encuentran la reforestación y la arborización de diferentes áreas del Valle... (Alcaldía de Medellín y Área Metropolitana del Valle de Aburrá, 2011; Ministerio de Ambiente y Desarrollo Sostenible, 2017). En particular, el Área Metropolitana creó el Plan de Siembra Aburrá para plantar un millón de árboles hasta 2019, a través de diferentes programas con la vinculación de la comunidad, los alcaldes metropolitanos, EPM, colectivos ambientales, las comunidades de los territorios, instituciones públicas y privadas. Desde el 2016 se han sembrado especies de árboles nativos como el Guayacán Amarillo y Rosado, Aguacatillo, Balso, Búcaro, Samán, Cámbulos, entre otras (AMVA, 2019).

Arroyave et al. (2019) identificó para el año 2015 una remoción de 46 toneladas de PM2.5 por árboles urbanos en el Valle de Aburrá, por medio del modelo i-Tree Eco, para ello se enviaron datos de calidad del aire y meteorológicos del 2014 al Servicio Forestal de Estados Unidos, los cuales administran la plataforma, sin estimar efectos marginales. Actualmente no hay ninguna herramienta disponible para calcular y proyectar la eliminación de contaminantes del aire por los árboles en esta región. 
En virtud de contribuir a la solución del problema de contaminación atmosférica en la ciudad, en esta investigación de enfoque cuantitativo, se estimó el efecto marginal que tendría el posible aumento de la cobertura arbórea urbana en el Valle de Aburrá sobre la remoción de PM2.5, para lo cual se utilizó una versión espacialmente distribuida del modelo UFORE. En particular, se consideró el impacto de aumentar o disminuir en un $1 \%$ el área sembrada, así como el impacto de incrementar la densidad de la vegetación a través del índice de área foliar (IAF).

\section{MATERIALES Y MÉTODOS}

\section{Área de estudio}

En la figura 1 se muestra el Valle de Aburrá y los municipios que lo conforman, así como la ubicación de las estaciones de calidad del aire (tabla 1 ) que se tuvieron en cuenta para el análisis de la situación actual, a las cuales se les asigna un área de influencia por medio de la técnica de Polígonos de Thiessen (figura 3).

Figura 1. El Valle de Aburrá y las estaciones de medición de PM2.5.

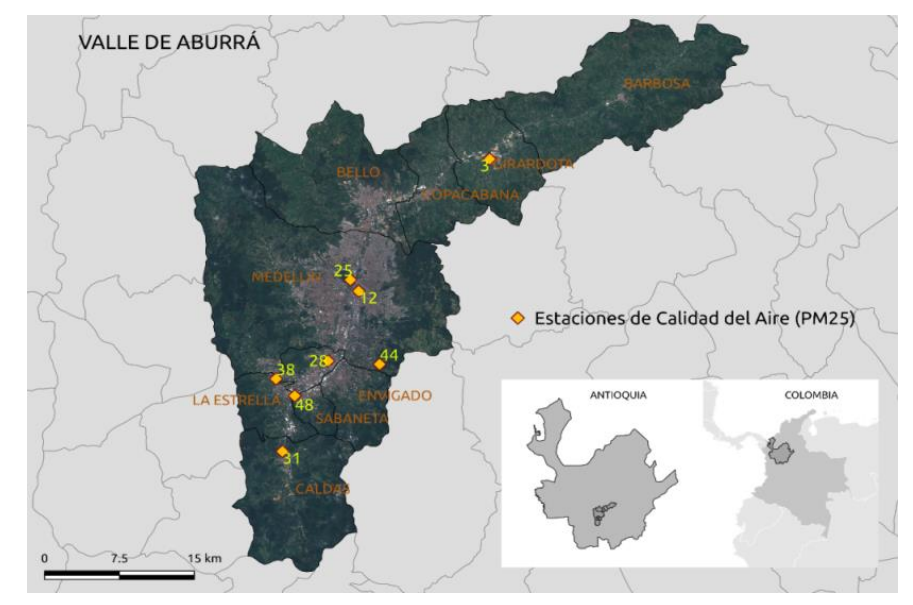

Fuente: Elaboración propia.

Tabla 1. Estaciones de Monitoreo de Calidad del Aire.

\begin{tabular}{|c|c|c|c|c|c|c|}
\hline Número & Longitud & Latitud & Nombre & Código & Longitud & Latitud \\
\hline 3 & -75.45091 & 6.37904 & $\begin{array}{l}\text { SOS Aburra Norte - } \\
\text { Girardota } \\
\text { Universidad Nacional de }\end{array}$ & GIR-SOS & -75.45091 & 6.37904 \\
\hline 25 & -75.57700 & 6.26337 & Colombia & $\begin{array}{l}\text { MED-UNNV } \\
\text { MED- }\end{array}$ & -75.57700 & 6.26337 \\
\hline 12 & -75.56958 & 6.25256 & Estación Tráfico Centro & MANT & -75.56958 & 6.25256 \\
\hline 44 & -75.55064 & 6.18254 & Tanques La YE & MED-LAYE & -75.55064 & 6.18254 \\
\hline 28 & -75.59721 & 6.18567 & $\begin{array}{l}\text { Casa de Justicia Itagüí } \\
\text { Colegio Concejo de }\end{array}$ & ITA-CJUS & -75.59721 & 6.18567 \\
\hline 38 & -75.64436 & 6.16850 & Itagüí & ITA-CONC & -75.64436 & 6.16850 \\
\hline 31 & -75.63863 & 6.09908 & Corporación Lasallista & CAL-LASA & -75.63863 & 6.09908 \\
\hline 48 & -75.62749 & 6.15231 & Estación Tráfico Sur & EST-METR & -75.62749 & 6.15231 \\
\hline
\end{tabular}

Fuente: Sistema de Alerta Temprana de Medellín y el valle de Aburrá (2019). 


\section{Cálculo de la remoción de material particulado}

Para realizar el cálculo de la remoción de PM2.5 se creó un código en lenguaje Python, siguiendo la metodología para la deposición seca de contaminantes del modelo UFORE, planteada por Nowak et al. (2013). El flujo horario de remoción de contaminantes por la vegetación se estima con la siguiente ecuación:

$$
F=V_{d} \cdot C \quad \text { Ecuación } 1 .
$$

Donde:

- $V_{d}$ Velocidad de deposición del contaminante en la superficie de la hoja

- C: Concentración de contaminante

En la figura 2 se presenta un esquema gráfico del modelo UFORE en general.

Figura 2. Esquema modelo componente deposición seca UFORE.

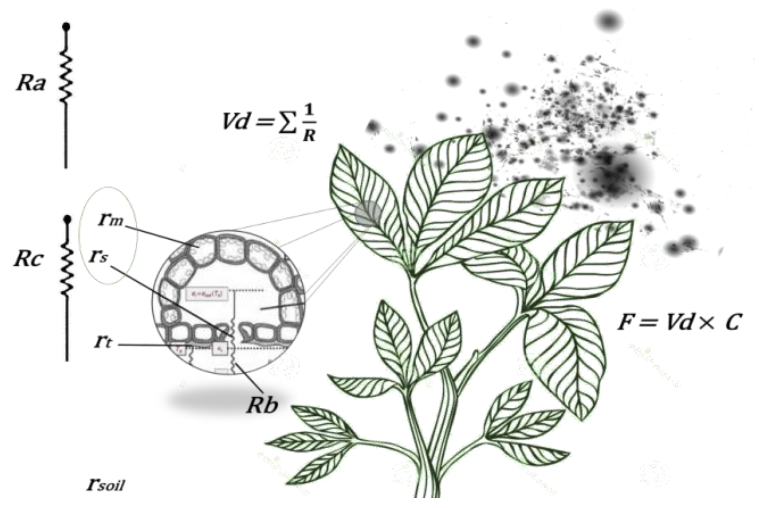

Nota: En el caso de contaminantes gaseosos $V_{d}$ es calculado como el inverso de la suma del inverso de la resistencia aerodinámica $(R a)$, cuasilaminar $(R b)$ y del dosel ( $R c)$. En el caso de contaminantes de material particulado, $V_{d}$ depende de la velocidad del viento. Fuente: Elaborado a partir de Hirabayashi et al. (2011) y Nowak et al. (2013).

La remoción se calculó a nivel horario, acumulada para todo el año 2017, con base en los registros de contaminación y meteorológicos suministrados por el Sistema de Alerta Temprana de Medellín y el Valle de Aburrá (SIATA). Los datos faltantes se imputaron con el método de la media móvil. En la figura 2, se presenta el ciclo promedio diario para cada estación.

Las velocidades de deposición (que varían en función de la velocidad del viento) y la resuspensión de PM2.5 en los árboles se estimaron a partir de la literatura (Freer-Smith et al., 2004; Beckett et al., 2000; y Pullman, 2009), siguiendo el trabajo de Nowak et al. (2013). Los valores de flujo se acumularon cada hora con un porcentaje del PM2.5 acumulado total sobre las horas actuales y anteriores, resuspendidas a la atmósfera según la velocidad del viento local. Durante los eventos de precipitación, el modelo asume que el PM2.5 acumulado, se lava de la superficie dependiendo de la magnitud de la lluvia. El flujo anual a las hojas de los árboles se estimó como el PM2.5 total lavado de estas durante el año, más la cantidad restante al final del año. La remoción se calcula con la siguiente ecuación:

$$
R=F \cdot A \quad \text { Ecuación } 2
$$


Donde:

- R es la remoción de contaminación

- F es el flujo contaminante

- A es la cobertura arbórea

Figura 3. Ciclo diario promedio de las concentraciones horarias de PM2.5 en el año 2017.

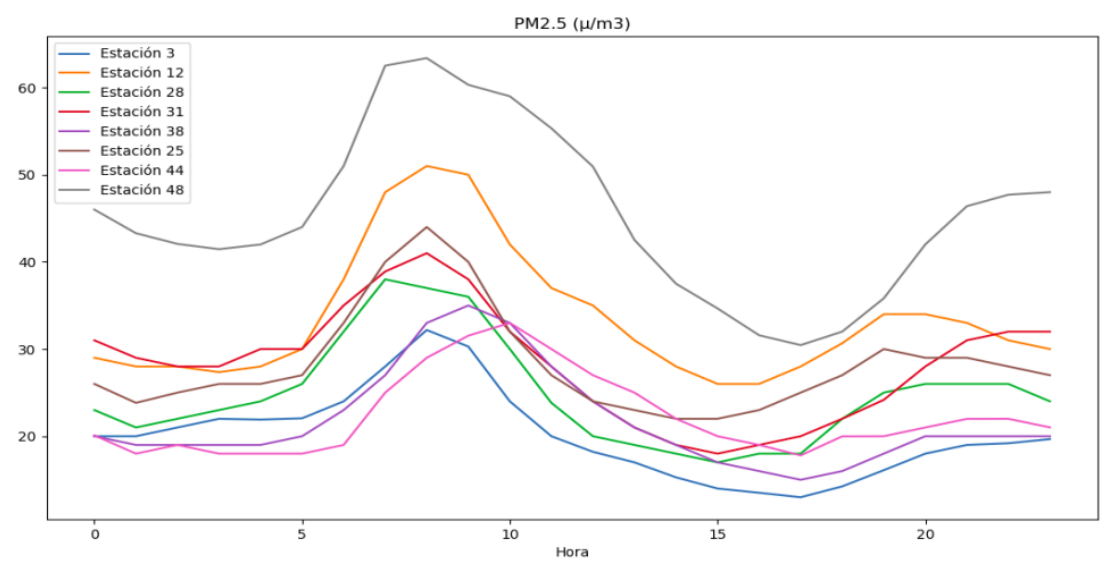

Fuente: Elaboración propia.

\section{Cálculo del IAF}

Para estimar el IAF se identificaron primero las coberturas arbóreas con imágenes satelitales Sentinel 2, utilizando la plataforma de Google Earth Engine (GEE). Se hizo un filtro de las imágenes con menor nubosidad para el año 2017; luego se promedió las imágenes para este año y se calculó el valor del NDVI. El IAF se calculó entonces con la siguiente ecuación:

$$
I A F=0.54+(9.96 \cdot N D V I) \quad \text { Ecuación } 3
$$

Para validar los rangos de valores de IAF a partir del análisis de imágenes satelitales se utilizó el inventario de la siembra y el seguimiento forestal, que proporcionó el Sistema de Árbol Urbano (SAU), administrado por la Secretaría de Medio Ambiente de Medellín.

\section{RESULTADOS Y DISCUSIÓN}

\section{Valores de IAF}

La figura 3 muestra los valores medios de IAF que se obtuvieron para el año 2017, como se explicó en la metodología. Se identificó una cobertura de árboles total de 99128.04 hectáreas, con IAF entre cuatro y nueve, en todo el Valle... 
Figura 4. Valores de IAF para el Valle de Aburrá y áreas arborizadas (ha), según Polígonos de Thiessen por estación de medición.

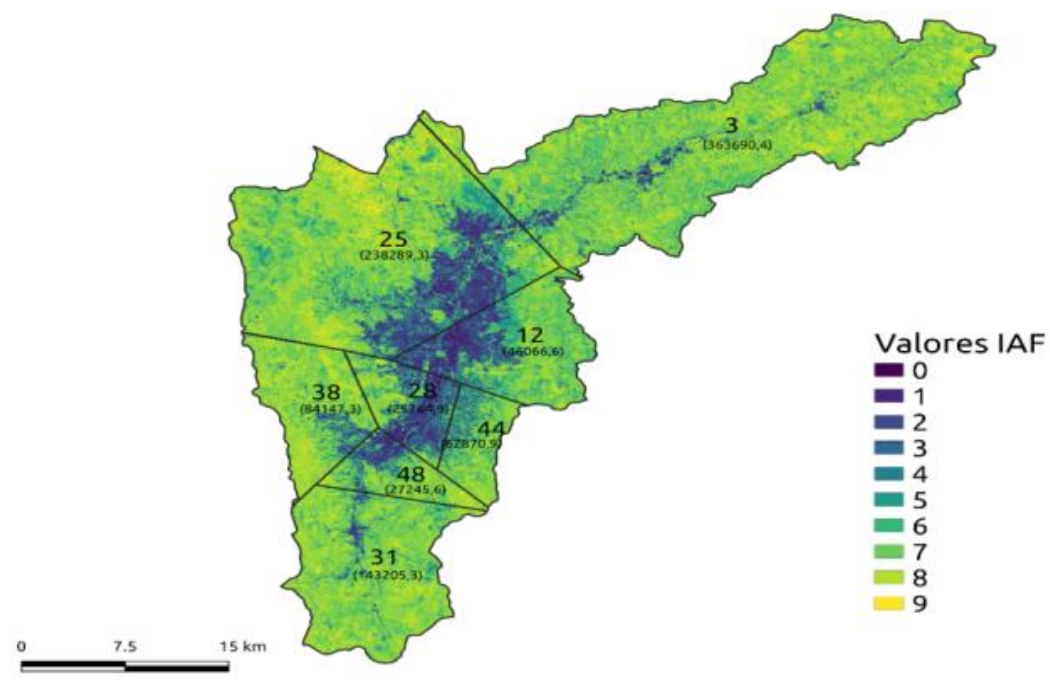

Fuente: Elaboración propia.

\section{Remociones de PM2.5 en el área metropolitana}

En la tabla 2 se presentan los resultados de la estimación del modelo UFORE para diferentes condiciones de cobertura arbórea.

Tabla 2. Remoción de material particulado en diferentes condiciones.

\begin{tabular}{lccc}
\hline \multicolumn{1}{c}{ Condición } & $\mathbf{R}$ (t/año) & \% AR ** & \% A Ar *** \\
\hline Base (2017) & 81.645 & 0 & 0 \\
Inicial + 1\% & 82.461 & 1 & 1 \\
Inicial + 1\% ZC & 82.977 & 1.63 & 1 \\
Inicial + Oriental & 81.646 & 0.001 & 0.002 \\
Inicial + ZSC & 82.367 & 0.88 & 0.85 \\
Inicial + ZSC (8) & 82.289 & 0.79 & 0.85 \\
& & & \% R Ar **** \\
Inicial con IAF 8 & 73.4 & -10.1 & 33.38 \\
Inicial con IAF 4 & 150.57 & 84.42 & 2.21 \\
\hline
\end{tabular}

Nota: *Remoción de PM2.5. ** Porcentaje que aumenta la remoción con respecto a la inicial. *** Porcentaje en que aumenta cobertura arbórea respecto a la inicial. ${ }^{* * *}$ Porcentaje que representa el IAF inicialmente en la cobertura arbórea. Fuente: Elaboración propia.

Primero se calculó la remoción de PM2.5 estimada para los niveles de contaminación y cobertura arbórea con el IAF de base del año 2017. En total, se obtuvo una remoción anual de 81.64 t. En el Valle... se tiene un inventario de emisiones actualizado para el 2016 (Área Metropolitana del Valle de Aburrá y Universidad Pontificia Bolivariana, 2018); al asumir que los valores se conservan para 2017, el porcentaje de remoción es del 4.37\%.

Cuando se aumenta el área total arborizada en un $1 \%$ de manera proporcional en toda el área metropolitana, sin modificar los valores de IAF, la remoción aumenta a $82.46 \mathrm{t}$, es decir, un incremento del $1 \%$ con respecto a la remoción inicial. Por otro lado, cuando se localiza ese $1 \%$ de 
aumento de la cobertura arbórea en las zonas de mayor concentración del contaminante, la remoción total es de 82.98 t, la cual representa un aumento de $1.63 \%$ en la remoción.

A partir de los avances que se han dado en las siembras de árboles en el Valle... (Plan de Siembra) se ha determinado que en el área urbana no hay espacios suficientes para esta acción, por tanto, la mayoría de las siembras se han dado en la zona rural y alrededor de aquel.

Un ejemplo de una siembra de árboles reciente fue el de la avenida Oriental en Medellín (figura 4), en 2018. Esta arborización, que representa un $0.001 \%$ del aumento en el área arbórea, ha tenido cierto impacto en el imaginario de los ciudadanos, aunque la remoción adicional que genera esta zona si está completamente arborizada y con un IAF óptimo de ocho, que es el valor máximo más común para los árboles inventariados en la base de datos de la Secretaría de Medio Ambiente (2018), sería solo de 904.93 gramos, lo que representa un aumento de $0.002 \%$ sobre la remoción inicial.

Dado lo anterior, en las imágenes satelitales se identificaron y ubicaron zonas que estuvieran sin arbolado y que se pudieran arborizar, lo cual representó un aumento del $0.85 \%$ del área arbórea de 2017, distribuidas sobre el Valle... (figura 4). Primero, se incrementó la arborización en dichas zonas con un IAF proporcional a los que existen en 2017, dando como resultado una remoción de $82.37 \mathrm{t}$. Luego, se aumentó esta arborización con un IAF de ocho, lo cual arrojó una remoción 82.29 t.

Por otro lado, para determinar el efecto de la densidad de vegetación se proyectó que todas las zonas con arbolado urbano del año 2017 tuvieran un IAF máximo de ocho, o que fueran árboles que pudieran alcanzarlo como, por ejemplo, Bauhiniapicta, Terminaliacatappa. En este caso, la remoción sería de 73.40 t, lo que representa una disminución de alrededor de un 10.10\% sobre la remoción base. También se proyecta que todas las zonas tuvieran árboles con un IAF mínimo de cuatro (pudiera ser Erythrina fusca). En este caso, la remoción aumentó a 150.57 t, lo cual representa un aumento de $84.42 \%$ sobre la remoción base.

Con los cálculos anteriores se esperaba que, con el aumento de la densidad del arbolado, la remoción de PM2.5 aumentara, sin embargo, ocurrió lo contrario. Esto podría explicarse analizando el modelo, pues este calcula la remoción en función del lavado que produce la lluvia por encima de un umbral en función del IAF (0.2 IAF); asimismo la resuspensión depende del viento sobre las hojas, por tanto, las especies de alto valor de IAF (óptimo de ocho) en la cobertura arbórea no serían tan efectivas como las del IAF en la condición actual, mientras que un IAF más bajo (mínimo de 4) sí representaría un aumento. No obstante, el modelo de deposición seca no tiene en cuenta las características de las hojas (algunas tienen vellosidades o ceras que pueden afectar la resuspensión y acumulación de partículas), ni considera una capacidad máxima de almacenamiento de PM2.5 en las estas. 
Figura 4. Áreas sin cobertura y cobertura proyectada en la avenida Oriental.
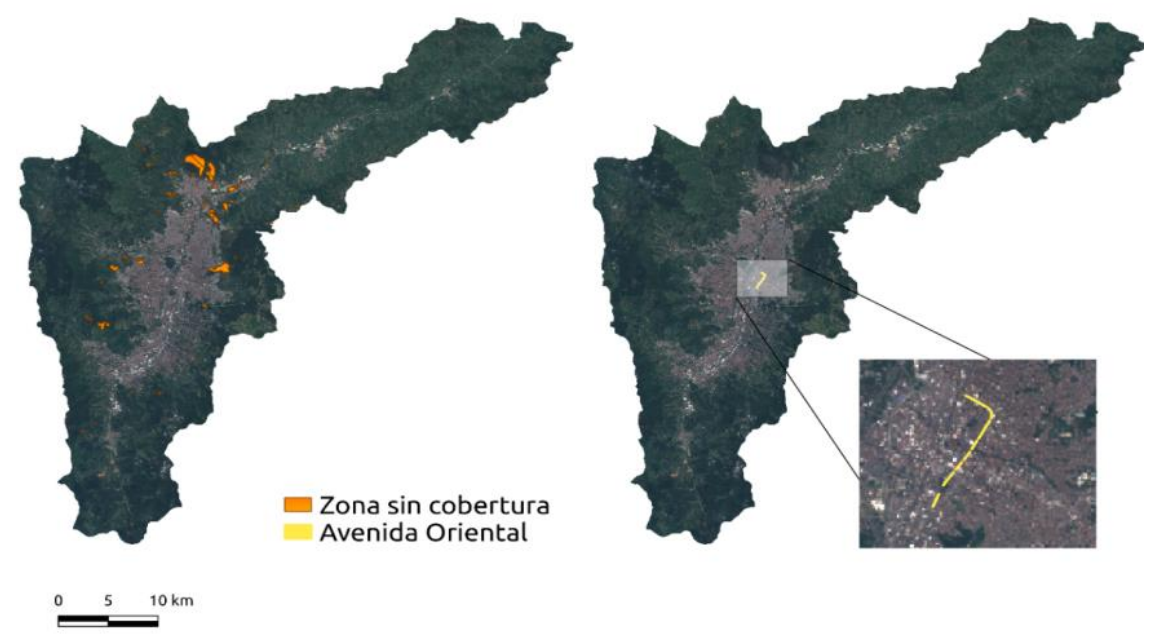

Fuente: Elaboración propia.

\section{Remoción de PM2.5 en Medellín}

Al calcular la remoción de PM2.5 para la zona urbana del municipio Medellín se presentó una remoción de $3.02 \mathrm{t}$. Cuando se aumenta toda el área arbórea de la ciudad en 1\%, la remoción aumenta en $1.3 \%$. Si el aumento se concentra en una zona con mayor contaminación, aumenta la remoción en un $2 \%$.

\section{CONCLUSIONES}

El modelo utilizado en este trabajo permite estimar los efectos marginales de remoción de contaminación atmosférica teniendo en cuenta al menos dos condiciones del arbolado: Su distribución espacial y su densidad de hojas. Estos efectos marginales son un aporte en la medida en que permitirán mejorar la planeación de las siembras dirigidas a mejorar la calidad del aire, como es el caso actual de Medellín.

Aunque la remoción de PM2.5 que se estimó con el modelo UFORE para la cobertura arbórea del Valle de Aburrá representa un pequeño porcentaje del flujo contaminante total (menos del 1\%), es innegable que representa un beneficio adicional a otras medidas de mitigación de los efectos de la contaminación del aire por material particulado.

De los cálculos preliminares se concluye que la densidad de hojas juega un papel importante en la magnitud de la remoción seca. Aparentemente, un valor bajo de IAF sería más efectivo en la remoción de PM2.5 que garantizar un valor alto en la cobertura arbórea. Sin embargo, el modelo no tiene en cuenta otras características del dosel y las hojas que podrían afectar este resultado. Además, la planeación de las siembras debe hacerse también considerando otros beneficios que ofrecen los árboles como la remoción de $\mathrm{CO}_{2}$, que requiere árboles con mayor biomasa, o la disminución de la temperatura ambiental en ciudades tan urbanizadas como Medellín, lo que representaría una mejora en la circulación atmosférica y en el bienestar de sus ciudadanos.

Como un aporte práctico para los gestores de decisión de la ciudad, este trabajo muestra que es probable que las siembras que se proyectan no representen un aumento substancial en la remoción de contaminación por PM2.5, al menos en el corto plazo, por tanto, es importante tener en cuenta otras medidas de mitigación para este margen de tiempo. 
Actualmente, la contaminación atmosférica por PM2.5 es la mayor problemática en el Valle..., sin embargo, es importante evaluar también el efecto que tienen los árboles en la remoción de otros contaminantes como $\mathrm{CO}, \mathrm{NOx}$ y ozono para estimar más beneficios derivados del arbolado. Igualmente, el modelo UFORE permite evaluar otros aspectos de la vegetación (composición de especies, densidad arbórea y numerosas funciones derivadas) que no fueron objeto de análisis en esta investigación, pero que pueden incidir en los resultados.

\section{REFERENCIAS BIBLIOGRÁFICAS}

Agudelo, L.C. y Miralles, J.L. (2015). Design and management of the metropolitan green belt of Aburrá Valley, Colombia. WIT Transactions on Ecology and the Environment, 194, 193-203. https://doi.org/10.2495/SC150181

Alcaldía de Medellín y Área Metropolitana Valle de Aburrá. (2011). Bio 2030 - Plan Director Medellín, Valle de Aburrá. (1ra ed.). Editorial Mesa Editores. https://n9.cl/i3te

Área Metropolitana del Valle de Aburrá y Universidad Pontificia Bolivariana. (2018). Inventario de emisiones atmosféricas del Valle de Aburrá - Año 2018. https://n9.cl/d3kux

Área Metropolitana del Valle de Aburrá. (2019). www.metropol.gov.co

Arroyave, M., Posada, M., Nowak, D. y Hoehn, R. (2019). Remoción de contaminantes atmosféricos por el bosque urbano en el valle de Aburrá. Colombia Forestal, 22(1), 5-16. http://dx.doi.org/10.14483/2256201X.13695

Beckett, K.P., Freer, P.H. y Taylor, G. (2000). Particulate pollution capture by urban trees: effect of species and windspeed. Global Change Biology, 6(8), 995-1003. https://doi.org/10.1046/j.1365-2486.2000.00376.x

Contraloría General de Medellín y Universidad Nacional de Colombia. (2018). Cuantificación física y económica del impacto de la contaminación atmosférica en salud de la población de la ciudad de Medellín. https://n9.cl/7y2i

Freer-Smith, P.H., El-Khatib, A.A. y Taylor, G. (2004). Capture of particulate pollution by trees: a comparison of species typical of semi-arid areas (Ficusnitida and Eucalyptus globulus) with European and North American species. Water, Air, and Soil Pollution, 155, 173-187. https://doi.org/10.1023/B:WATE.0000026521.99552.fd

Herrera, L. (2015). Caracterización de la Capa Límite Atmosférica en el valle de Aburrá a partir de la información de sensores remotos y radiosondeos [Tesis de Maestría, Universidad Nacional de Colombia]. http://www.bdigital.unal.edu.co/51042/1/1128283242.2015.pdf

Hirabayashi, S., Kroll, C. N., y Nowak, D. J. (2011). Urban Forest Effects-Dry Deposition (UFORE-D) Model Descriptions, 1-23. https://cutt.ly/7yDnsWr

Jeanjean, A.P.R., Buccolieri, R., Eddy, J., Monks, P. S. y Leigh, R.J. (2017). Air quality affected by trees in real street canyons: The case of Marylebone neighbourhood in central London. Urban Forestry \& Urban Greening, 22, 41-53. https://doi.org/10.1016/j.ufug.2017.01.009

Ministerio de Ambiente y Desarrollo Sostenible. (2017). Pronunciamiento situación calidad del aire de Medellín [Video]. https://n9.cl/d8ga 
Nowak, D.J., Hirabayashi, S., Bodine, A. y Hoehn, R. (2013). Modeled PM2.5 removals by trees in ten U.S. cities and associated health effects. Environmental Pollution, 178, 395-402. https://doi.org/10.1016/j.envpol.2013.03.050

Pullman, M. (2009). Conifer PM2.5 Deposition and Re-suspension in Wind and Rain Events [Master's thesis, Cornell University]. https://ecommons.cornell.edu/handle/1813/11239

Rendón, A., Wirth, V., Salazar, J.F., Palacio, C.A. \& Brötz, B. (December 14 to 18, 2014). Mechanisms of Air Pollution Transport in Urban Valleys as a Result of the Interplay Between the Temperature Inversion and the Urban Heat Island Effect. In AGU Fall Meeting Abstracts. https://ui.adsabs.harvard.edu/abs/2014AGUFM.B1310327R/abstract

Reynolds, C., Escobedo, F., Clerici, N. y Zea-Camaño, J. (2017). Does "Greening" of Neotropical Cities Considerably Mitigate Carbon Dioxide Emissions? The Case of Medellin, Colombia. Sustainability, 9(5), 785. https://doi.org/10.3390/su9050785

Rogers, K., Sacre, K., Goodenough, J., \& Kieron Doick. (2015). Valuing London's Urban Forest: results of the London i-Tree Eco Project. Treeconomics London. https://n9.cl/1ua4

Secretaría de Medio Ambiente (2018). Base de datos del Sistema de Árbol Urbano. https://www.medellin.gov.co/sau/

Selmi, W., Weber, C., Rivière, E., Blond, N., Mehdi, L. y Nowak, D. (2016). Air pollution removal by trees in public green spaces in Strasbourg city, France. Urban Forestry \& Urban Greening, 17(1), 192-201. https://doi.org/10.1016/j.ufug.2016.04.010

Sistema de Alerta Temprana de Medellín y el valle de Aburrá. (2019). https://siata.gov.co/siata nuevo/ 